\title{
The long-term course of temporal lobe epilepsy: from unilateral to bilateral interictal epileptiform discharges in repeated video EEG monitorings
}

Stephanie Gollwitzer ${ }^{1,3}$, Catherine A. Scott ${ }^{1}$, Fiona Farrell ${ }^{1,2}$, Gail S. Bell ${ }^{1,2}$, Jane de Tisi ${ }^{1}$,

Matthew C. Walker ${ }^{1}$, Tim Wehner ${ }^{1}$, Josemir W. Sander ${ }^{1,2,4}$, Hajo M. Hamer ${ }^{3}$, Beate Diehl ${ }^{1}$

${ }^{1}$ NIHR University College London Hospitals Biomedical Research Centre, UCL Institute of

Neurology, Queen Square, London, WC1N 3BG, United Kingdom

${ }^{2}$ Epilepsy Society, Chalfont St Peter, SL9 0RJ United Kingdom

${ }^{3}$ Epilepsy Center, Department of Neurology, University Hospital Erlangen, Erlangen, Germany

${ }^{4}$ Stichting Epilepsie Instellingen Nederland (SEIN), Heemstede, The Netherlands

Corresponding author: Stephanie Gollwitzer, Epilepsy Center, Department of Neurology University Hospital Erlangen, Schwabachanlage 6, 91054 Erlangen, Phone: +49(0)91318544525, Email: Stephanie.gollwitzer@uk-erlangen.de 


\begin{abstract}
Introduction: Bilateral interictal epileptiform discharges (IED) and ictal patterns are common in temporal lobe epilepsy (TLE) and have been associated with decreased chances of seizure freedom after epilepsy surgery. It is unclear whether secondary epileptogenesis, although demonstrated in experimental models, exists in humans and may account for progression of epilepsy.
\end{abstract}

Material and Methods: We reviewed consecutive video EEG recordings from 1992 to 2014 repeated at least two years apart (mean interval 6.14 years) in 100 people with epilepsy diagnosed with TLE.

Results: Ictal EEG patterns and IEDs remained restricted to one hemisphere in 36 people (group 1), 46 exhibited bilateral abnormalities from the first recording (group 2), 18 progressed from unilateral to bilateral EEG pathology over time (group 3). No significant differences between the three groups were seen with respect to age at epilepsy onset, duration, or underlying pathology. Extra-temporal IED during the first EEG recording were associated with an increased risk of developing bilateral epileptiform changes over time (hazard ratio $3.67 ; 95 \%$ CI $1.4,9.4$ ).

Conclusion: Our findings provide some support of progression in TLE and raise the possibility of secondary epileptogenesis in humans. The development of an independent contra-lateral epileptogenic focus is known to be associated with a less favourable surgical outcome. We defined reliable EEG markers for an increased risk of progression to more widespread or independent bitemporal epileptogenicity at an early stage, thus allowing for individualized pre-surgical counselling.

Key words: Focal epilepsy, Temporal lobe epilepsy, Secondary epileptogenesis, Electroencephalography 


\section{INTRODUCTION}

Temporal lobe epilepsy (TLE) is the commonest pharmacoresistant focal epilepsy in humans. $[1,2]$ Over $50 \%$ of people with TLE become medically refractory over time.[3-5] Epilepsy surgery provides a $60 \%$ chance of long-term seizure freedom (Engel class 1) in well-selected surgical candidates and should therefore be considered early in the course of the condition.[69] Negative predictors for surgical outcome include older age at surgery, longer duration of epilepsy and the presence of generalized tonic-clonic seizures,[10] whereas interictal epileptiform discharges (IED) restricted to the operated temporal lobe correlate positively with postoperative seizure freedom. $[11,12]$

Independent bilateral IED are reported in 40-60\% of individuals with TLE[13, 14] and are commonly regarded as the electrophysiological correlate of bitemporal excitability.[15]

Several studies have investigated the correlation between bilateral IED, epilepsy duration and seizure frequency. It has been hypothesized that bilaterality in TLE is the result of an ongoing process of broadening of the epileptogenic network resulting eventually in independent seizure foci, a concept referred to as secondary epileptogenesis.[16, 17] Results have been controversial; some researchers found progressive epileptiform EEG changes over time[18] while others reported the opposite.[17, 19]

Studies in animal models of unilaterally induced seizures have shown that an epileptogenic mirror focus can occur in the homotopic area of the contra-lateral hemisphere.[20-23]

Systematic longitudinal analyses of clinical and electrophysiological changes in human TLE are lacking and it remains unclear whether people with TLE are at risk of disease progression, which may lead to decreased chances of seizure freedom following surgery. Predictors for impending secondary epileptogenesis that could guide individual counselling with respect to 
the natural course of their epilepsy and their expected surgical outcome at different time points are also unknown.

We hypothesized that in some people with TLE, the condition progresses resulting in an increased bilaterality of independent interictal epileptiform activity with or without bilaterality of ictal onset zones. Such an evolution would provide some electrophysiological evidence for secondary epileptogenesis in people with TLE.

\section{MATERIAL AND METHODS}

\section{Patients}

We included individuals with presumed TLE aged at least 18 who had more than one prolonged video EEG recording with an interval of at least two years between the first and the last and no epilepsy surgery in between. The assumed epileptogenic zone had to be located in one temporal lobe based on semiology, EEG, or imaging data. People whose TLE was associated to progressive pathology were excluded.

Between January 1992 and June 2014, 2796 individuals underwent presurgical long-term scalp EEG recordings at the National Hospital for Neurology and Neurosurgery (Queen Square and Chalfont sites). Of these, 1167 (42\%) were diagnosed with TLE and 345 (30\%) of these had more than one video EEG monitoring session lasting typically between 5 and 7 days. One hundred of these fulfilled the inclusion criteria and were reviewed. Demographic data and clinical characteristics were obtained from clinical notes, as well as surgical outcomes for those who eventually proceeded to surgery $(\mathrm{N}=31)$. Imaging and pathology results were reviewed from the hospital database. 
The exercise was ethically approved as part of an audit into evaluation of outcome and utilisation of video EEG services over time.

\section{EEG analysis}

EEG was recorded digitally at a sampling rate of $256 \mathrm{~Hz}$. For review, filter settings were kept at 0.5 to $70 \mathrm{~Hz}$ bandpass and sensitivity at $150 \mu \mathrm{V} / \mathrm{cm}$. All had at least 19 electrodes coverage following the international 10-20-system, 68 had additional left and right superficial sphenoidal electrodes $(\mathrm{Sp} 1,2)$ and closely spaced electrodes according to the international 10-10-system over both temporal regions.

\section{Interictal EEG}

To evaluate interictal epileptic abnormalities, we reviewed the first 24 hours of recording, prior to any seizure provoking measures, e.g. drug reduction or sleep deprivation and, whenever possible, prior to spontaneous seizures. If seizures occurred during the first 24 hours of recording (17 recordings), an alternative seizure-free 24 hour interval was reviewed. If no such interval was available, a one hour period post-ictally was excluded from the analysis (5 recordings). Sleep and wake states were evaluated separately. Epileptiform interictal activities (spikes, sharp waves, and polyspikes) were identified based on generally accepted criteria.[24]

For localization and mapping of the maximum negativity of the spike or sharp wave, we analyzed the upstroke using a referential montage against the contra-lateral occipital electrode $(\mathrm{O} 2, \mathrm{O} 1)$. We allocated each discharge to an anatomic region based on its maximum negativity. Electrodes Sp1/2, F7/8, F9/10, FT7/8, FT9/10 were referred to as anterior temporal, electrodes T7/8, T9/10, TP7/8, TP9/10, P7/8 as lateral temporal and the rest of the scalp electrodes as extra-temporal.[25, 26]

\section{Ictal EEG}


All recorded seizures were reviewed in an anterior-posterior bipolar montage, incorporating additional temporal and sphenoidal electrodes when present and in a referential montage against an indifferent electrode for amplitude measurements.

Ictal patterns were characterized as: rhythmic activity in the alpha, theta or delta frequency range; paroxysmal fast (rhythmic activity $>13 \mathrm{~Hz}$ ); suppression (activity $<10 \mu \mathrm{V}$ in amplitude); repetitive epileptiform activity ( $\geq 3$ discharges in sequence); arrhythmic activity (irregular, mixed frequency waveforms).

The distribution of ictal patterns was classified as:[27]

1. Focal anterior or lateral temporal: activity maximal at a single electrode with no more than two adjacent electrodes within $80 \%$ to $100 \%$ of the maximal electrode

2. Regional temporal: activity equally involving two or more electrodes of one temporal lobe

3. Lateralized: activity involving multiple electrodes over two or more lobes of a single hemisphere

4. Bitemporal: activity involving both temporal lobes equally

5. Generalized / non-lateralized: activity involving multiple electrodes over both hemispheres equally

6. Non-localizable

Ictal patterns had to appear within 30 seconds after clinical seizure onset to be of lateralizing or localizing value. Otherwise the seizure was labelled "non-localizable".

\section{Case categorization}

Epileptiform activity was defined as "unilateral" if all ictal patterns were ipsi-lateral to the epileptogenic lesion (if present) or best hypothesis of the epileptogenic zone (EZ) (distribution classification 1, 2 and 3). Also, 100\% of IED had to be unilateral and ipsi-lateral to the assumed side of the EZ. To be referred to as "bilateral epileptiform", an EEG recording 
had to fulfil one or more of the following criteria: IED appeared bilateral or contra-lateral to the epileptogenic lesion or in non-lesional cases to the assumed hemisphere of epileptogenicity based on clinical, EEG, and neuropsychological data; seizures were accompanied by a generalized, non-localizable or bi-temporal pattern (distribution classification 4, 5, and 6); different seizures originated from both temporal lobes independently; seizures originated from the temporal lobe contra-lateral to the epileptogenic lesion. Accordingly, we categorized people into three groups: Group 1 included individuals whose EEG showed exclusively unilateral epileptic activity during all reviewed recordings. People who already exhibited bilateral epileptic activity in the first recording were assigned to Group 2. Group 3 consisted of those who showed strictly unilateral during the first but bilateral epileptic activity during one or more subsequent recordings.

For analysis of ictal patterns, each individual was characterised as having seizures of "focal/regional", "lateralised", or "bilateral/generalised" origin. Those with focal/regional onset had all ictal patterns focal or regional, whereas those categorised as lateralised had at least $25 \%$ of seizures lateralised with the remainder focal/regional, while those with bilateral/generalised onset had at least $25 \%$ of seizures in this category.

\section{Statistical analysis}

We used non-parametric tests to compare continuous variables (as most had skewed distributions) and exact tests to compare categorical variables. We performed univariate analysis to compare clinical and epidemiological data between the three groups. EEG factors affecting the risk of developing bilateral epileptiform changes over time were determined using cox regression unless proportionality assumptions were not valid in which case log rank tests were carried out. Cox regression analysis was used to compare time to becoming bilateral in groups 1 and 3; in group 1 time was censored at the time of the last recording, 
whereas in group 3, time to failure was the time of the first EEG with bilateral features. For comparison of surgical outcome we used Fisher's exact test.

\section{RESULTS}

Of the 100 people reviewed, $51 \%$ were female and $86 \%$ right-handed. The age of seizure onset ranged between 1 and 42 years (median 13.5). For details on clinical and epidemiologic data see table 1 . In all cases a decision was made on the predominant side of epileptogenicity based on clinical, EEG, neurophysiology or imaging data. In 80 an unequivocal lesion was seen on MRI (37 in the right temporal lobe, 43 in the left; hippocampal sclerosis (HS) in 62).

The ictal and interictal EEG of 36 remained stable with exclusively unilateral IED and ictal onset patterns (group 1). The EEG of 46 people (group 2) showed bilateral epileptiform activity from the first EEG recording. Two of these exhibited only unilateral IED during a later recording, while all others in this group remained bilateral throughout. Group 3 consisted of 18 individuals with progression from unilateral to bilateral IED and / or ictal patterns over time.

We found no significant differences between the three groups regarding age at disease onset, epilepsy duration, history of febrile seizures, number of anticonvulsants tried, or MRI pathology (table 1).

While the frequency of focal seizures at the time of the first EEG recording was similar in all three groups $(\mathrm{p}=0.32)$, there was a trend towards higher frequency of generalized seizures in group 3 compared with group 1 although the difference was not significant $(p=0.06)$.

Thirty-one people eventually proceeded to surgery and all had lesions on MRI. Histopathological examination confirmed the assumed pathology in all. We found no 
statistically significant difference between the three groups in terms of post-surgical outcome (table 2).

To define early EEG markers for subsequent progression of epileptiform activity to the contra-lateral, formerly unaffected hemisphere, we compared the first EEG recordings of groups 1 and 3. As we could not clarify whether bilateral epileptic activity in group 2 was present from disease onset or had developed from unilateral involvement prior to the first recording, group 2 was excluded from this part of the analysis.

In group 1, IED during the first recording were mostly restricted to sphenoidal and anterior temporal electrodes. Spikes and sharp waves with an extra-temporal maximum were very rare, appearing in only one of 36 people $(2.8 \%)$, whereas a significantly higher percentage of individuals $(8 / 18 ; 44.4 \%)$ in group 3 exhibited extra-temporal IED during their first VT. Cox regression showed that the presence of extra-temporal unilateral IED in the first recording was associated with a higher probability of developing bilateral epileptiform activity (i.e. being in group 3). Lateral temporal IED were also less common in group 1 (9/32, 28.1\%) compared with group $3(13 / 18,72.2 \%)$, but the log rank test showed no significant difference. At the time of the second recording 2 of $36(5.6 \%)$ people in group 1 and 10/18 (55.6\%) in group 3 had extra-temporal IED, and 13 of 33 (39\%) in group 1 and 12 of $18(67 \%)$ in group 3 had lateral temporal IED. For overall IED distribution see table 3.

59 people had a seizure during the first recording, 18 in group A and 8 in group C. Of 19 people whose seizure onset was focal or regional, 15 remained in group A while of seven with lateral seizure onset only three remained in group A. Lateral ictal onset at the first recording was associated with a slightly higher probability of developing bilateral epileptiform activity than was focal or regional ictal onset (HR 2.61, 95\% CI 0.65, 10.44). 


\section{DISCUSSION}

The existence and relevance of secondary epileptogenesis in human focal epilepsy has been long debated.[28] It is assumed that seizure-induced neuronal death and associated fibre degeneration leads to mossy fibre sprouting and synaptic reorganization thus forming recurrent excitatory circuits. This is regarded as the underlying pathological mechanism. Loss of hilar neurons and their influence on inhibitory inter-neurons then make those inter-neurons "dormant" thus dis-inhibiting a large population of cells even in areas distant to the injured site.[29-31] This causes structural alterations in remote areas that receive projections from the primary focus.

Earlier studies investigated whether the concept of secondary epileptogenesis derived from animal models could be translated to human focal epilepsy. Only two studies have reviewed serial EEGs over time, one reporting that the EEG remains mainly stable [19] while the other found a yearly increase in the incidence of bilateral IED of about $1 \%$ [18]. We found that a considerable number of people with TLE progress from initially unilateral towards bilateral disease which might support the existence of secondary epileptogenesis. An alternative underlying mechanism could be the maturation of a "proepileptic" lesion, a mechanism recently discussed as an explanation of late postsurgical failure.[32] We cannot rule out that contralateral tissue generating seizures in the later course has been potentially epileptogenic from the beginning and that the limited EEG sampling time was not sufficient to detect bilateral abnormalities. Very few of those with bilateral abnormalities were found to have unilateral abnormalities at a later date and this militates against this explanation.

We did not find any significant differences between people with stable unilateral and progressive TLE in terms of epilepsy duration or age at disease onset suggesting that pathology progression is a non-linear, active process in susceptible individuals rather than a simple function of elapsing time. Reasons for susceptibility, however, are still unclear. It has 
been stated that epileptogenicity is variable in different brain areas and lesion types. Bilateral IED have been reported to be more common in mesial than in neocortical temporal lobe epilepsy[33] while posterior or extra-temporal IED have been associated with mesial temporal tumors rather than HS.[25]

HS, the most common pathology in our cohort, was present in the same frequency in stable and progressive disease. A strong correlation between seizure frequency and the likelihood of developing a secondary focus earlier has been reported.[34] Conversely, a more recent study found no differences in terms of seizure frequency between "temporal plus" epilepsies, involving temporal and extra-temporal networks and people with "pure" TLE.[35] We observed no significant differences in the frequency of focal seizures between the three groups. There was, however, a trend towards a higher total number of seizures in group three compared to group 1. This finding would be in line with the observations of Morrell, who stated that the development of a secondary focus becomes more likely with an increasing number of seizures. [34] These results stress the importance of rigorous seizure control and, if possible, early surgical intervention.

Generalized seizures were slightly more frequent amongst those with signs of progression (group 3) than amongst those showing stable unilateral disease. As patients in both groups received mainly broad-spectrum AED with good efficacy on generalized seizures, divergent therapeutic regimes do not explain this finding. The groups did also not differ in terms of comorbidities, such as migraine or traumatic head injuries, that could account for the effect. The inability to restrict seizures to a focal region could be the clinical correlate of a preexistent widespread network of pathologic circuits thus promoting not only seizure spread but also enabling secondary epileptogenesis. Conversely, generalized seizures could create pathways for pathology spread, thus causing susceptibility to develop secondary foci in primarily unsusceptible individuals.[29] 
Specific white matter changes could be the structural basis for pathology spread as described in a recent diffusion tensor imaging study that found callosal fibers of the tapetum significantly more damaged in those with bilateral IED.[36]

The lack of statistically significant differences in terms of seizure outcome after surgery between the three groups may be attributable to the low statistical power, as only 31 people underwent surgery. The small number of people proceeding to surgery eventually might be due to a selection bias in our cohort, as those undergoing repeated VT may represent a subgroup with difficult-to-assess epilepsies leading to more hesitant recommendations for surgery and lower estimated odds for postoperative seizure freedom. Risks to cognition and choices and attitudes to surgery vary, and may also explain the low surgical uptake.

Our findings are of clinical relevance, as people should be informed about the natural course of their condition including potential risk for progression. Whether this means they will have a worse prognosis after epilepsy surgery requires further investigations, but seems likely.[11, 12, 37-39] Prerequisite for such an individualized counselling approach is the definition of reliable markers for early prediction of later pathology spread.

Our results show a strong correlation between the presence of extra-temporal unilateral IED on baseline EEG and bilateral IED on subsequent recordings (HR 3.7). Assuming that secondary epileptogenesis is an ongoing process, the manifestation of IED outside the diseased temporal lobe might indicate a propensity for epileptogenicity to involve remote areas and eventually the contra-lateral hemisphere. Concordant with this assumption, seizures showing widespread lateralized ictal patterns were more common in people who later developed contra-lateral involvement than in those with a stable unilateral disease. 
There are, however, several limitations. Firstly, our cohort was heterogeneous in terms of underlying pathology. The sample size of specific lesions other than HS was not large enough to analyze results separately. Secondly, although neither the overall seizure frequency nor the average IED frequency assessed differed significantly between the intra-individual EEG recordings, we cannot exclude that changes in epilepsy activity might have influenced our results in single cases. Lastly, although we aimed to analyze interictal data in a truly seizurefree interval being aware that frequency and distribution of IED are often stated to be influenced by preceding seizures [40] a complete 24 hour seizure free interval was not always available.

\section{CONCLUSION}

Our findings provide some support for the existence of secondary epileptogenesis in human TLE. It has to be assumed that the presence of an independent contra-lateral epileptogenic focus decreases the chances of postoperative seizure freedom. Epilepsy surgery should be considered earlier rather than later in the course of the disease. The ictal and interictal scalp EEG provides reliable markers for an increased risk of pathology spread at an early stage thus permitting to identify those at risk and allowing for individualized counselling. 


\section{Acknowledgements}

This work was undertaken at UCLH/UCL, which received a proportion of funding from the Department of Health's NIHR Biomedical Research Centres funding scheme.

\section{Conflicts of Interest}

Gail Bell's family has shares in GlaxoSmithKline. Josemir Sander receives research support from the Dr. Marvin Weil Epilepsy Research Fund, Eisai, GlaxoSmithKline, WHO, and EU FP7 and has been consulted by and received fees for lectures from GSK, Eisai, Lundbeck, Teva and UCB. The remaining authors have no conflicts of interest. 


\section{References}

[1]Helmstaedter C, May TW, von Lehe M, Pfaefflin M, Ebner A, Pannek HW, et al. Temporal lobe surgery in Germany from 1988 to 2008: diverse trends in etiological subgroups. Eur J Neurol 2014;21: 827-34.

[2]Engel J, Jr. Mesial temporal lobe epilepsy: what have we learned? Neuroscientist 2001;7: 340-52.

[3] Semah F, Picot MC, Adam C, Broglin D, Arzimanoglou A, Bazin B, et al. Is the underlying cause of epilepsy a major prognostic factor for recurrence? Neurology 1998;51: 1256-62.

[4]Stephen LJ, Brodie MJ. Surgery for temporal-lobe epilepsy. N Engl J Med 2002;346: 2925 .

[5]Dhamija R, Moseley BD, Cascino GD, Wirrell EC. A population-based study of long-term outcome of epilepsy in childhood with a focal or hemispheric lesion on neuroimaging. Epilepsia 2011;52: 1522-6.

[6]Engel J, Jr. Surgery for seizures. N Eng1 J Med 1996;334: 647-52.

[7]Wiebe S, Blume WT, Girvin JP, Eliasziw M. A randomized, controlled trial of surgery for temporal-lobe epilepsy. N Engl J Med 2001;345: 311-8.

[8]de Tisi J, Bell GS, Peacock JL, McEvoy AW, Harkness WF, Sander JW, et al. The longterm outcome of adult epilepsy surgery, patterns of seizure remission, and relapse: a cohort study. Lancet 2011;378: 1388-95.

[9]Wiebe S, Jette N. Pharmacoresistance and the role of surgery in difficult to treat epilepsy. Nat Rev Neurol 2012;8: 669-77.

[10]Janszky J, Janszky I, Schulz R, Hoppe M, Behne F, Pannek HW, et al. Temporal lobe epilepsy with hippocampal sclerosis: predictors for long-term surgical outcome. Brain 2005;128: 395-404.

[11]Hennessy MJ, Elwes RD, Rabe-Hesketh S, Binnie CD, Polkey CE. Prognostic factors in the surgical treatment of medically intractable epilepsy associated with mesial temporal sclerosis. Acta Neurol Scand 2001;103: 344-50.

[12]Radhakrishnan K, So EL, Silbert PL, Jack CR, Jr., Cascino GD, Sharbrough FW, et al. Predictors of outcome of anterior temporal lobectomy for intractable epilepsy: a multivariate study. Neurology 1998;51: 465-71.

[13]Ergene E, Shih JJ, Blum DE, So NK. Frequency of bitemporal independent interictal epileptiform discharges in temporal lobe epilepsy. Epilepsia 2000;41: 213-8. 
[14]Williamson PD, French JA, Thadani VM, Kim JH, Novelly RA, Spencer SS, et al. Characteristics of medial temporal lobe epilepsy: II. Interictal and ictal scalp electroencephalography, neuropsychological testing, neuroimaging, surgical results, and pathology. Ann Neurol 1993;34: 781-7.

[15]Steinhoff BJ, So NK, Lim S, Luders HO. Ictal scalp EEG in temporal lobe epilepsy with unitemporal versus bitemporal interictal epileptiform discharges. Neurology 1995;45: 889-96. [16]Gupta PC, Dharampaul, Pathak SN, Singh B. Secondary epileptogenic EEG focus in temporal lobe epilepsy. Epilepsia 1973;14: 423-6.

[17]Niediek T, Franke HG, Degen R, Ettlinger G. The development of independent foci in epileptic patients. Arch Neurol 1990;47: 406-11.

[18]Hughes JR. Long-term clinical and EEG changes in patients with epilepsy. Arch Neurol 1985;42: 213-23.

[19]Holmes MD, Dodrill CB, Wilkus RJ, Ojemann LM, Ojemann GA. Is partial epilepsy progressive? Ten-year follow-up of EEG and neuropsychological changes in adults with partial seizures. Epilepsia 1998;39: 1189-93.

[20]Morrell F, deToledo-Morrell L. From mirror focus to secondary epileptogenesis in man: an historical review. Adv Neurol 1999;81: 11-23.

[21]Morrell F, Bradley W, Ptashne M. Effect of drugs on discharge characteristics of chronic epileptogenic lesions. Neurology 1959;9: 492-8.

[22]Sobayo T, Mogul DJ. Rapid onset of a kainate-induced mirror focus in rat hippocampus is mediated by contralateral AMPA receptors. Epilepsy Res 2013;106: 35-46.

[23]Wilder BJ. The mirror focus and secondary epileptogenesis. Int Rev Neurobiol 2001;45: 435-46.

[24]Cascino GD, Trenerry MR, So EL, Sharbrough FW, Shin C, Lagerlund TD, et al. Routine EEG and temporal lobe epilepsy: relation to long-term EEG monitoring, quantitative MRI, and operative outcome. Epilepsia 1996;37: 651-6.

[25]Hamer HM, Najm I, Mohamed A, Wyllie E. Interictal epileptiform discharges in temporal lobe epilepsy due to hippocampal sclerosis versus medial temporal lobe tumors. Epilepsia 1999;40: 1261-8.

[26]Diehl B, Najm I, Mohamed A, Babb T, Ying Z, Bingaman W. Interictal EEG, hippocampal atrophy, and cell densities in hippocampal sclerosis and hippocampal sclerosis associated with microscopic cortical dysplasia. J Clin Neurophysiol 2002;19: 157-62.

[27]Foldvary N, Klem G, Hammel J, Bingaman W, Najm I, Luders H. The localizing value of ictal EEG in focal epilepsy. Neurology 2001;57: 2022-8. 
[28]Scholly J, Valenti MP, Staack AM, Strobl K, Bast T, Kehrli P, et al. Hypothalamic hamartoma: is the epileptogenic zone always hypothalamic? Arguments for independent (third stage) secondary epileptogenesis. Epilepsia 2013;54 Suppl 9: 123-8.

[29]Sloviter RS. The functional organization of the hippocampal dentate gyrus and its relevance to the pathogenesis of temporal lobe epilepsy. Ann Neurol 1994;35: 640-54.

[30]Wuarin JP, Dudek FE. Electrographic seizures and new recurrent excitatory circuits in the dentate gyrus of hippocampal slices from kainate-treated epileptic rats. J Neurosci 1996;16: 4438-48.

[31]Salin P, Tseng GF, Hoffman S, Parada I, Prince DA. Axonal sprouting in layer V pyramidal neurons of chronically injured cerebral cortex. J Neurosci 1995;15: 8234-45.

[32]Najm I, Jehi L, Palmini A, Gonzalez-Martinez J, Paglioli E, Bingaman W. Temporal patterns and mechanisms of epilepsy surgery failure. Epilepsia 2013;54: 772-82.

[33]Pacia SV, Devinsky O, Perrine K, Ravdin L, Luciano D, Vazquez B, et al. Clinical features of neocortical temporal lobe epilepsy. Ann Neurol 1996;40: 724-30.

[34]Morrell F. Varieties of human secondary epileptogenesis. J Clin Neurophysiol 1989;6: 227-75.

[35]Barba C, Barbati G, Minotti L, Hoffmann D, Kahane P. Ictal clinical and scalp-EEG findings differentiating temporal lobe epilepsies from temporal 'plus' epilepsies. Brain 2007;130: 1957-67.

[36]Pustina D, Doucet G, Skidmore C, Sperling M, Tracy J. Contralateral interictal spikes are related to tapetum damage in left temporal lobe epilepsy. Epilepsia 2014;55: 1406-14.

[37]Jehi LE, Silveira DC, Bingaman W, Najm I. Temporal lobe epilepsy surgery failures: predictors of seizure recurrence, yield of reevaluation, and outcome following reoperation. $\mathbf{J}$ Neurosurg 2010;113: 1186-94.

[38]Schulz R, Luders HO, Hoppe M, Tuxhorn I, May T, Ebner A. Interictal EEG and ictal scalp EEG propagation are highly predictive of surgical outcome in mesial temporal lobe epilepsy. Epilepsia 2000;41: 564-70.

[39]Holmes MD, Dodrill CB, Ojemann GA, Wilensky AJ, Ojemann LM. Outcome following surgery in patients with bitemporal interictal epileptiform patterns. Neurology 1997;48: 103740.

[40]Janszky J, Fogarasi A, Jokeit H, Schulz R, Hoppe M, Ebner A. Spatiotemporal relationship between seizure activity and interictal spikes in temporal lobe epilepsy. Epilepsy Res 2001;47: 179-88. 



\section{Tables}

Table 1 Clinical, epidemiological and MRI characteristics of people with epilepsy and unilateral versus bilateral epileptiform activity

\begin{tabular}{|c|c|c|c|c|c|}
\hline & $\begin{array}{l}\text { group } 1^{\circ} \\
n=36\end{array}$ & $\begin{array}{l}\operatorname{group} 2^{\circ} \\
n=46\end{array}$ & $\begin{array}{l}\text { group } 3^{\circ \circ 0} \\
n=18\end{array}$ & $\begin{array}{l}\text { p group } \\
1,2,3\end{array}$ & $\begin{array}{l}\text { p group } \\
1 \& 3\end{array}$ \\
\hline $\begin{array}{l}\text { Male sex N (\%) } \\
(\mathrm{N}=100)\end{array}$ & $21(58.33)$ & $22(47.83)$ & $6(33.33)$ & 0.23 & 0.15 \\
\hline $\begin{array}{l}\text { Age at epilepsy onset* } \\
(\mathrm{N}=82)\end{array}$ & $15(9,24.5)$ & $10.5(2,20)$ & $12(9.5,23.5)$ & 0.13 & 0.77 \\
\hline $\begin{array}{l}\text { Epilepsy duration at } 1^{\text {st }} \mathrm{VT}^{*} \\
(\mathrm{~N}=82)\end{array}$ & $\begin{array}{l}16.5 \\
(10.5,28)\end{array}$ & $\begin{array}{l}19.5 \\
(12,32)\end{array}$ & $\begin{array}{l}16.5 \\
(10.5,24)\end{array}$ & 0.48 & 0.49 \\
\hline $\begin{array}{l}\text { Time between VTs*x } \\
(\mathrm{N}=100)\end{array}$ & $5(3,8)$ & $5.5(3,8)$ & $7(3,8)$ & 0.72 & 0.61 \\
\hline $\begin{array}{l}\text { Right handed N(\%) } \\
(\mathrm{N}=78)\end{array}$ & $\begin{array}{l}24 / 27 \\
(88.9)\end{array}$ & $\begin{array}{l}31 / 36 \\
(86.1)\end{array}$ & $\begin{array}{l}12 / 15 \\
(80.0)\end{array}$ & 0.53 & 0.57 \\
\hline $\begin{array}{l}\text { History of febrile convulsions } \\
N(\%) \quad(N=66)\end{array}$ & $\begin{array}{l}10 / 27 \\
(37.0)\end{array}$ & $8 / 26(30.8)$ & $\begin{array}{l}5 / 13 \\
(38.5)\end{array}$ & 0.84 & $>0.99$ \\
\hline $\begin{array}{l}\text { Number of AEDs tried * } \\
(\mathrm{N}=71)\end{array}$ & $7(5,8)$ & $8(6,10)$ & $7(5,10)$ & 0.34 & 0.66 \\
\hline $\begin{array}{l}\text { Hippocampal sclerosis } N(\%) \\
(\mathrm{N}=62)\end{array}$ & $\begin{array}{l}22 / 35 \\
(62.9)\end{array}$ & $\begin{array}{l}29 / 46 \\
(63.0)\end{array}$ & $\begin{array}{l}11 / 18 \\
(61.1)\end{array}$ & 0.99 & $>0.99$ \\
\hline $\begin{array}{l}\text { Side of epileptogenicity } \\
\mathrm{N}(\%) \text { right }(\mathrm{N}=100)\end{array}$ & $\begin{array}{l}12 / 36 \\
(33.3)\end{array}$ & $\begin{array}{l}17 / 46 \\
(37.0)\end{array}$ & $\begin{array}{l}8 / 18 \\
(44.4)\end{array}$ & 0.81 & 0.75 \\
\hline $\begin{array}{l}\text { Frequency of focal seizures } *^{\mathrm{xx}} \\
(\mathrm{N}=89)\end{array}$ & $4(2,12)$ & $7(3,12)$ & $8(4,12)$ & 0.32 & 0.17 \\
\hline $\begin{array}{l}\text { Frequency of generalized } \\
\text { seizures }{ }^{* x}(N=88)\end{array}$ & $0(0,0.3)$ & $0(0,1)$ & $0.2(0.05,1)$ & 0.16 & 0.06 \\
\hline \multicolumn{6}{|c|}{$\begin{array}{l}\text { group 1: people with unilateral interictal epileptic discharges and seizure patterns in all } \\
\text { recordings; }{ }^{\circ} \text { group 2: people with bilateral interictal epileptic discharges and/ or seizure patterns in all } \\
\text { recordings; }{ }^{\circ} \text { group 3: people with unilateral epileptic discharges and seizure patterns in the first and } \\
\text { bilateral interictal epileptic discharges and/ or seizure patterns in a consecutive recording. Univariate } \\
\text { analysis without correction for different factors, comparison between all groups and group } 1 \text { and 3; } \\
\text { *median, IQR; }{ }^{\mathrm{x}} \text { group } 1 \text { and } 2: \text { time between first and last EEG, group 3: time between last unilateral } \\
\text { and first bilateral EEG; years; }{ }^{\mathbf{x x}} \text { at time of } 1^{\text {st }} \text { recording per month }\end{array}$} \\
\hline
\end{tabular}


Table 2 Surgical outcome in groups 1, 2 and 3 after 1,2 and 3 years

\begin{tabular}{lllll}
\hline & $\begin{array}{l}\text { group 1 } \\
\mathbf{n = 1 2 / 3 6}\end{array}$ & $\begin{array}{l}\text { group 2 } \\
\mathbf{n = 1 5 / 4 6}\end{array}$ & $\begin{array}{l}\text { group 3 } \\
\mathbf{n = 4 / 1 8}\end{array}$ & $\mathbf{p}$ \\
\hline Seizure free year 1 $^{\mathrm{x}}$ & $8 / 12$ & $8 / 15$ & $3 / 4$ & 0.68 \\
Seizure free year 2 $^{\mathrm{x}}$ & $4 / 6$ & $6 / 14$ & $2 / 4$ & 0.85 \\
Seizure free year 3 $^{\mathrm{x}}$ & $4 / 6$ & $5 / 14$ & $2 / 4$ & 0.62 \\
\hline
\end{tabular}

Fisher's exact test; ${ }^{x}$ number of people with ILAE class 1 outcome 1, 2 and 3 years postoperatively 
Table 3 Distribution and localization of IED and ictal patterns at $1^{\text {st }}$ EEG recording

\begin{tabular}{|c|c|c|c|c|c|}
\hline & $\begin{array}{l}\text { group 1 } \\
n=36\end{array}$ & $\begin{array}{l}\text { group } 3 \\
n=18\end{array}$ & HR & $\begin{array}{l}95 \% \\
\text { CI }\end{array}$ & $\mathbf{P}$ \\
\hline PWE with extra-temporal IED N (\%) & $\begin{array}{l}1 / 36 \\
(2.8)\end{array}$ & $\begin{array}{l}8 / 18 \\
(44.4)\end{array}$ & 3.67 & $1.38,9.74$ & 0.01 \\
\hline PWE with lateral temporal IED N (\%) & $\begin{array}{l}9 / 32 \\
(28.1)\end{array}$ & $\begin{array}{l}13 / 18 \\
(72.2)\end{array}$ & & & $0.08^{\#}$ \\
\hline IED distribution in \%: & & & & & \\
\hline Maximum sphenoidal electrode & 68.8 & 35.2 & & & \\
\hline Maximum anterior temporal * & 89.7 & 60.9 & & & \\
\hline Maximum lateral temporal ** & 10. 02 & 34.0 & & & \\
\hline Maximum extra-temporal & 0.29 & 5.14 & & & \\
\hline $\begin{array}{l}\text { Seizure onset: } \\
\text { PWE with focal or regional ictal onset } \\
\text { compared with }\end{array}$ & $15 / 18$ & $4 / 8$ & 1 & & \\
\hline PWE with lateral ictal onset ${ }^{++}$ & $3 / 18$ & $4 / 8$ & 2.61 & $0.65,10.44$ & 0.18 \\
\hline
\end{tabular}

Comparison of ictal and interictal pathology in the first EEG recording between group 1 and 3, by definition no contra-lateral IED or ictal patterns are present in both groups at that time; Cox regression, HR: hazard ratio, PWE: people with epilepsy; CI: 95\% confidence interval; ${ }^{*} \log$ rank tests were performed due to lack of proportionality; *electrodes Sp1/2, F7/8, F9/10, FT7/8, FT9/10; ** electrodes T7/8, T9/10, TP7/8, TP9/10, P7/8; ${ }^{+} 100 \%$ of seizures with focal or regional onset; ${ }^{++} \geq 25 \%$ of seizures with lateralized onset 